\title{
LA JUSTICIA EUROPEA CONTRA LA REDUCCIÓN ARBITRARIA DE LA EDAD DE JUBILACIÓN DE LOS JUECES. COMENTARIO A LA SENTENCIA DEL TJUE DE 24 DE JUNIO DE 2019, COMISIÓN/POLONIA, C-619/18
}

\section{EUROPEAN JUSTICE AS THE LAST RESORT FOR THE POLISH JUDICIARY: CJEU AGAINST THE ARBITRARY LOWERING OF THE RETIREMENT AGE OF JUDGES. COMMENTARY ON THE JUDGMENT OF 24 JUNE 2019, COMMISSION V. POLAND, C-619/18}

\author{
Adam Krzywoń \\ Universidad de Varsouia \\ German Research Institute for Public Administration (FÖV), Speyer
}

Cómo citar / Nola aipatu: Krzywoń, A. (2020). La justicia europea contra la reducción arbitraria de la edad de jubilación de los jueces. Comentario a la sentencia del TJUE de 24 de junio de 2019, Comisión/Polonia, C-619/18. Legebiltzarreko Aldizkaria LEGAL - Revista del Parlamento Vasco, (1): 164-175. https://doi.org/10.47984/legal.2020.007

\section{RESUMEN}

En la Sentencia de 24 de junio de 2019, Comisión/Polonia (Independencia del Tribunal Supremo), C-6r9/ı8, EU:C:20I9:53I, el Tribunal de Justicia de la Unión Europea se pronunció sobre las normas de jubilación de los jueces del Tribunal Supremo polaco, declarando contrario al Derecho de la Unión el mecanismo que reducía de manera arbitraria la edad de jubilación. En el presente comentario se analizan los últimos cambios en el sistema judicial polaco y el contexto del fallo del TJUE. Sobre todo, el objeto de estudio es el cambio de la jurisprudencia europea y la evolución de instrumentos disponibles para las instituciones de la Unión Europea para vigilar por la independencia judicial. Se demuestran también los elementos de la independencia judicial indicados por el TJUE y las normas acerca de la jubilación de los jueces.

\section{PALABRAS CLAVE}

Independencia judicial, jubilación de los jueces, Unión Europea, Tribunal de Justicia de la Unión Europea, recurso de incumplimiento. 


\section{ABSTRACT}

In the judgment of 24 June 20I9, Commission v. Poland (Independence of the Supreme Court), C-619/18, EU:C:2019:53I, the Court of Justice of the European Union ruled on the retirement of Polish Supreme Court judges, declaring that the mechanism of arbitrarily lowering the retirement age was contrary to the European Union law. This commentary analyses the latest changes in the Polish judicial system and the context of the CJEU ruling. The study is focused in the change in European case-law and the development of instruments available to the EU institutions for monitoring judicial independence. The paper discusses also the elements of the judicial independence indicated by CJEU and the rules on the retirement of judges.

\section{KEYWORDS}

Judicial independence, retirement of judges, European Union, Court of Justice of the European Union, treatyinfringement proceeding.

\section{LABURPENA}

20I9ko ekainaren 24ko epaian, Batzordea/Polonia (Auzitegi Gorenaren independentzia), C-6I9/18, EU: c: 2019:53I epaian, Europar Batasuneko Justizia Auzitegiak Poloniako Auzitegi Goreneko epaileen erretiro-arauei buruzko iritzia eman zuen, eta erretiro-adina modu arbitrarioan murrizten zuen mekanismoa Batasunaren zuzenbidearen aurkakotzat jo zuen. Iruzkin honetan Poloniako sistema judizialaren azken aldaketak eta EBJAren epaiaren testuingurua aztertzen dira. Batez ere, aztergai da Europako jurisprudentziaren aldaketa eta Europar Batasuneko erakundeek independentzia judiziala zaintzeko eskura dituzten tresnen bilakaera. EBJAk adierazitako independentzia judizialaren elementuak eta epaileen erretiroari buruzko arauak ere frogatzen dira.

\section{GAKO-HITZAK}

Independentzia judiziala, epaileen erretiroa, Europar Batasuna, Europar Batasuneko Justizia Auzitegia, ez-betetze errekurtsoa. 
En la Sentencia de 24 de junio de 2019, Comisión/Polonia (Independencia del Tribunal Supremo), C-619/18, EU:C:2019:531, el Tribunal de Justicia de la Unión Europea (en adelante TJUE) se pronunció sobre las normas para la jubilación de los jueces del Tribunal Supremo polaco (en adelante TS). Declaró contrario al Derecho de la Unión el mecanismo que reducía la edad de jubilación de los jueces del TS (de 70 a 65 años) y permitía aplicar esta medida a los jueces en ejercicio nombrados antes de su entrada en vigor (3 de abril de 2018). Por otra parte, el TJUE rechazó la posibilidad de atribuir al órgano ejecutivo (Presidente de la República) la facultad discrecional para prorrogar la función jurisdiccional de los jueces del TS una vez alcanzada la nueva edad de jubilación. La sentencia del TJUE tiene mucha importancia no solo para la judicatura polaca, ya que establece el estándar mínimo de la independencia judicial en toda la Unión Europea. Por lo tanto, vale la pena analizar detalladamente el fallo del TJUE, mostrando primero el contexto de la sentencia, enfocándose luego en el procedimiento con que fue dictada (recurso de incumplimiento, art. 258 TFUE), y finalmente examinando los elementos esenciales de la independencia judicial indicados por el TJUE.

Hay que mencionar que no es la única sentencia dictada últimamente por el TJUE contra Polonia en el contexto del asalto al Poder judicial. Los recientes cambios legislativos sirvieron de catalizador para elaborar principios relativos al estatus del juez europeo. Se trata entonces de una serie de dictámenes compuesta por las sentencias de 25 de julio de 2018, Minister for Justice and Equality (Deficiencias del sistema judicial), C-216/18 PPU, EU:C:2018:586; 24 de junio de 2019, Comisión/Polonia (Independencia del Tribunal Supremo), C-619/18, EU:C:2019:531; 5 de noviembre de 2019, Comisión/Polonia (Independencia de los Tribunales Ordinarios), C-192/18, EU:C:2019:924 y 19 de noviembre de 2019, A.K. (Independencia de la Cámara Disciplinaria del Tribunal Supremo), asuntos acumulados C-585/18, C-624/18 y C-625/18, EU:C:2019:982. Para el presente análisis, vale la pena destacar sobre todo la sentencia Comisión/Polonia (Independencia de los Tribunales Ordinarios), dado que el TJUE analizaba en ella unos instrumentos muy parecidos al caso de los jueces del TS. También se trataba de la jubilación precipitada de los jueces (reducción de la edad de jubilación de 67 a 60 años en el caso de las mujeres, de 67 a 65 años en el caso de los hombres) y la posibilidad de decidir sobre la prórroga de las funciones por un órgano ejecutivo (en este caso, el Ministro de Justicia).

Hay que mencionar también que en el momento de dictar la Sentencia Comisión/ Polonia (Independencia del Tribunal Supremo), de 24 de junio de 2019, las disposiciones controvertidas de la ley polaca habían sido derogadas. Fue la consecuencia de una resolución provisional adoptada por el TJUE el 17 de diciembre de 2018, en la que se ordenó la suspensión de la reducción de la edad de jubilación y se obligó a las autoridades polacas a adoptar todas las medidas necesarias para que los jueces del 
TS afectados pudiesen continuar ejerciendo sus funciones en los mismos puestos y disfrutando de los mismos derechos, condiciones de empleo y estatuto que antes. La medida provisional provocó un cambio de la legislación (desde el 1 de enero de 2019), ya que quedaron derogadas las disposiciones que reducían la edad de jubilación de los jueces y las normas que facultaban al Presidente de la República a autorizar la prórroga de las funciones jurisdiccionales. A todos los jueces de entre 65 y 70 años se les facultó para volver al estado activo. Al mismo tiempo, el legislador polaco aplicó un mecanismo de presión económica. Debido a que el regreso a las funciones jurisdiccionales no era obligatorio, los jueces podían quedar jubilados conservando el $100 \%$ de su remuneración, mientras que en condiciones normales es el $75 \%$. Una parte de los jueces de entre 65 y 70 años, cansados de los constantes ataques políticos, han decidido seguir jubilados.

Además, el gobierno polaco, deseando evitar que el TJUE declare definitivamente el incumplimiento de las obligaciones del Derecho de la Unión, alegaba que, debido a los mencionados cambios legislativos, el procedimiento ante el TJUE había quedado sin objeto. Estos argumentos no convencieron al TJUE, ya que, según la jurisprudencia anterior, se considera que la existencia del incumplimiento debe ser apreciada en función de la situación del Estado miembro tal como esta se presentaba en el momento de iniciar el procedimiento, y los cambios ocurridos posteriormente no pueden ser tomados en cuenta ${ }^{1}$.

II.

Los últimos cambios en el sistema judicial polaco forman parte de un fenómeno que en la doctrina actual se denomina constitutional populism o democratic backsliding (véanse p.ej. Szente, 2017: 456-475, Blokker 2019: 519-543 y Sadurski, 2019). Se trata de ataques frontales contra todas las agencias públicas independientes, sobre todo contra la estabilidad y la autonomía del Poder judicial, con el fin de subordinar el funcionamiento de los tribunales al factor político (Castillo-Ortiz, 2019: 48-72 y Faggiani, 2019: 57-100). Se utilizan varias técnicas legislativas con el propósito de destituir a los jueces (Brzozowski, 2018: 275-292). Simultáneamente observamos el fenómeno de court-packing, que consiste en incrementar el número de jueces, permitiendo a la mayoría política de turno presentar sus candidatos y controlar el proceso de su nombramiento (Chang, 2019: 456-457). Otro método, importante desde el punto de vista del presente análisis, es la manipulación de la edad de jubilación de los jueces. La mayoría parlamentaria en Polonia aplicó todas estas medidas y desde 2015 logró, por etapas, paralizar varios sectores del Poder judicial (Krzywoń, 2018:

1 Sentencia Comisión/Polonia (Independencia del Tribunal Supremo), apdo. 30-31. 
359-379). Todo empezó con la captura del Tribunal Constitucional con el fin de convertirlo en un órgano dispuesto a legitimar los controvertidos cambios legislativos (Brzozowski, 2019: 371-382 y Garlicki, 2019: 141-162). Las etapas siguientes fueron el asalto a la independencia del TS, el Consejo Nacional del Poder Judicial y los tribunales ordinarios (Sadurski, 2019: 97-126).

La modificación de la edad de jubilación de los jueces polacos, tanto de los tribunales ordinarios como del TS, era posible sin una reforma constitucional. La ley fundamental polaca no indica claramente ninguna norma sustancial, exigiendo solamente que la edad de jubilación se determine en la ley ordinaria (art. 180 apdo. 4). Al hacerlo, el legislador está sin duda obligado a respetar una serie de principios constitucionales (entre ellos, independencia judicial y separación de poderes), pero frente al mencionado silencio constitucional, y en el actual ambiente político, estas normas no se tomaron en cuenta.

Los cambios controvertidos consistían en la modificación de las normas de la ley ordinaria del TS de 2002, que fijaba la edad de jubilación de los jueces en 70 años. Las nuevas normas rebajaron esta edad a 65 años, permitiendo a los interesados presentar, antes de cumplirlos, una declaración en la que indicaban su deseo de continuar ejerciendo sus funciones. Sobre la prórroga - de tres años, con la posibilidad de renovarla una vez, también por tres años- decidía el Presidente de la República, después de haber solicitado un dictamen, puramente formal, del Consejo Nacional del Poder Judicial. Hay que enfatizar que la reducción de la edad de jubilación se refería también a los jueces que hubieran alcanzado los 65 años en la fecha de entrada en vigor de las nuevas normas. De un día para otro, los jueces entre 65 y 70 años perdieron el estatus de juez en funciones, aunque también tenían la posibilidad de solicitar al Presidente de la República la autorización para continuar desempeñando su cargo de juez.

Según los argumentos del gobierno polaco, estas modificaciones se debían a la voluntad de armonizar la jubilación de los jueces con la edad general de jubilación, aplicable al conjunto de los trabajadores, y de optimizar así la estructura por edades de los miembros del TS con responsabilidades directivas ${ }^{2}$. En este contexto vale la pena mencionar que en el 2017 se ha rebajado la edad de jubilación en Polonia, fijándola en 60 años para las mujeres y 65 años para los hombres. Esta modificación, contraria a las tendencias europeas y mundiales, despierta serias dudas en relación con la prohibición de la discriminación por razón de sexo³ .

2 Sentencia Comisión/Polonia (Independencia del Tribunal Supremo), apdo. 80

3 Por este motivo, en la sentencia Comisión/Polonia (Independencia de los Tribunales Ordinarios), el TJUE, apoyándose en el art. 157 TFUE, rechazó toda diferenciación en materia de retribución entre jueces y juezas, cualquiera que sea el mecanismo que determine esta desigualdad. La fijación de un requisito de edad distinto según el sexo para la concesión de una pensión constitutiva de retribución, según el TJUE, es contraria al Derecho de la Unión, debido a que se trata de condiciones directamente discriminatorias por razón de sexo (véase apdo. 74-80). 
Las disposiciones mencionadas afectaron con carácter inmediato a 27 de los 72 jueces del TS que estaban en activo en la fecha de entrada en vigor de la nueva ley, entre ellos a su Presidenta Primera. En este contexto se produjo otro problema constitucional, debido a que la Constitución establece expressis verbis un mandato del Presidente Primero del TS de seis años (art. 183 apartado 3). La reducción de la edad de jubilación significaba entonces no solo la injerencia en la independencia judicial, sino también la modificación de la norma constitucional a través de la ley ordinaria ${ }^{4}$.

III.

Declarar el incumplimiento del Derecho de la Unión y la violación del principio de la independencia judicial no hubiera sido posible sin un importante cambio en la jurisprudencia europea, iniciado por la Sentencia de 27 de febrero de 2018, Associação Sindical dos Juízes Portugueses, C-64/16, EU:C:2018:117. El TJUE presentó una nueva interpretación de la obligación del art. 19 TUE, apdo. 1, párrafo segundo, de establecer un sistema de vías de recurso que garantice la tutela judicial efectiva en los ámbitos cubiertos por el Derecho de la Unión. Para cumplir con ello hay que garantizar que los órganos judiciales nacionales -que potencialmente pueden pronunciarse sobre la aplicación o la interpretación del Derecho de la Unión- gocen siempre de la independencia judicial, que forma parte del contenido esencial del derecho fundamental a un juicio justo (art. 47 de la Carta de los Derechos Fundamentales de la Unión Europea, en adelante CDFUE) $)^{5}$. Elemento muy importante de este razonamiento es la presunción de que los tribunales nacionales pueden tener que resolver sobre cuestiones relacionadas con el Derecho de la Unión ${ }^{6}$. Lo anterior significa que el TJUE rompe con el principio que hasta entonces determinaba su facultad para pronunciarse en un asunto determinado: se exigía la existencia de un vínculo sustancial entre el Derecho de la Unión y la legislación nacional, para evitar la extensión de las competencias de la UE a los asuntos reservados a los poderes nacionales.

En efecto, desde la sentencia Associação Sindical dos Juízes Portugueses, el TJUE está siempre habilitado para examinar la independencia de los tribunales nacionales (Krzywoń, 2020: 94-96). En primer lugar, corresponde a los Estados miembros determinar la organización del sistema de la Administración de Justicia, pero no es menos cierto que, al ejercer esta competencia frente al Poder judicial, siempre deben

4 Según la Constitución, el mandato de la Presidenta Primera debía finalizar el 30 de abril de 2020 y, como efecto de la nueva ley, se produjo el día de su entrada en vigor: 3 de abril de 2018; véase sentencia Comisión/Polonia (Independencia del Tribunal Supremo), apdo. 62.

5 Sentencia Associação Sindical dos Juízes Portugueses, apdo. 32-34.

6 Sentencia Comisión/Polonia (Independencia del Tribunal Supremo), apdo. 42-59 
cumplir con las obligaciones que les impone el Derecho de la Unión ${ }^{7}$. Al exigir que los Estados miembros cumplan con el estándar mínimo de independencia judicial, ni la UE ni el TJUE pretenden en modo alguno ejercer por sí mismos dicha competencia ni, por tanto, arrogársela. En consecuencia, los Estados miembros organizan su Administración de Justicia bajo la supervisión europea (Bonelli y Claes, 2018: 630).

Debido al mencionado cambio de jurisprudencia, hay que destacar la evolución de otros instrumentos del Derecho de la Unión, sobre todo el recurso de incumplimiento (art. 258 TFUE), que fue utilizado por la Comisión Europea para cuestionar la reducción de la edad de jubilación de los jueces del TS. Es posible alegar que un sistema nacional no cumple con los requisitos de la independencia de los jueces, integrada en el contenido del derecho a un proceso equitativo y tutela judicial efectiva (art. 19 TUE, apdo. 1, párrafo segundo, y art. 47 CDFUE). Al mismo tiempo, la Comisión puede solicitar el procedimiento acelerado ${ }^{8}$ y la adopción de las medidas provisionales ${ }^{9}$, tal como hizo en el caso de los jueces del TS polaco.

Vale la pena resaltar que el procedimiento previsto en el art. 258 TFUE permite al TJUE evaluar directamente las disposiciones nacionales, no -como en el supuesto de las cuestiones prejudiciales (art. 267 TFUE)- indicar tan solo la interpretación correcta del Derecho de la Unión. Además, aunque el TJUE no tiene competencias para eliminar las normas del ordenamiento nacional, el art. 260 TFUE formula expresamente la obligación del Estado miembro de adoptar las medidas necesarias para la ejecución de la sentencia que declare el incumplimiento del Derecho de la Unión, por lo cual asume parcialmente el papel de un actor de la constitucionalidad (Martín y Pérez de Nanclares, 2017: 265). En el caso de que el Estado no ejecute la sentencia, es posible también imponerle el pago de una suma a tanto alzado o de una multa coercitiva. Gracias a todo esto, como se ha evidenciado por la reacción del gobierno polaco al mencionado auto del TJUE de 17 de diciembre de 2018 sobre las medidas provisionales, estos instrumentos pueden ser muy eficaces a la hora del control europeo directo de si la legislación nacional cumple con el estándar de la independencia judicial en la UE.

7 Sentencias Associação Sindical dos Juízes Portugueses, apdo. 40, y Comisión/Polonia (Independencia del Tribunal Supremo), apdo. 52.

8 Véase art. 133, apdo. 1, del Reglamento del Procedimiento del Tribunal de Justicia.

9 Véase art. 160, apdo. 2 y 7, del Reglamento del Procedimiento del Tribunal de Justicia. 
IV.

En la sentencia Comisión/Polonia (Independencia del Tribunal Supremo), el TJUE se ha pronunciado sobre la noción de la independencia judicial. La relaciona con las garantías del sistema europeo de derechos humanos, indicando que debe velar por que la interpretación del art. 47, párrafo segundo, CDFUE garantice el mismo nivel de protección que el art. 6 del Convenio Europeo de Derechos Humanos, según lo interpreta el Tribunal Europeo de Derechos Humanos ${ }^{10}$. Indica además que la independencia de los órganos jurisdiccionales aglutina dos aspectos ${ }^{11}$. El primero, de orden externo, supone que el órgano en cuestión ejerza sus funciones con plena autonomía, sin estar sometido a ningún vínculo jerárquico o de subordinación y sin recibir órdenes ni instrucciones de ningún tipo, de tal modo que quede protegido de injerencias o presiones externas que puedan hacer peligrar la independencia de sus miembros a la hora de juzgar, o que puedan influir en sus decisiones. Esta indispensable libertad frente a los elementos externos exige ciertas garantías, como p.ej. la inamovilidad, lo que significa que los jueces pueden permanecer en el ejercicio de sus funciones en tanto no hayan alcanzado la edad de jubilación forzosa o hasta que termine su mandato, cuando este tenga una duración determinada. Aunque no tiene carácter absoluto, este principio solo puede ser objeto de excepciones cuando existan motivos legítimos e imperiosos que lo justifiquen, y siempre que se respete el principio de proporcionalidad ${ }^{12}$.

El segundo aspecto de la independencia -de orden interno- se asocia al concepto de imparcialidad y se refiere a la equidistancia que debe guardar el órgano de que se trate con respecto a las partes del litigio y a sus intereses respectivos en relación con el objeto de dicho litigio. En la doctrina, esta dimensión de la independencia se denomina imparcialidad subjetiva, para enfatizar que los jueces no deberían ser prejuzgados personalmente (Lautenbach, 2013: 164). Se exige entonces el respeto del principio de objetividad y la inexistencia de cualquier interés en la solución del litigio que no sea el de la interpretación y aplicación estricta de la norma jurídica. Sin embargo, en la práctica jurisprudencial resulta muy difícil separar estos dos órdenes de la independencia, ya que por independencia interna se entiende también toda protección de los jueces frente a los ataques que provienen desde dentro de la esfera del Poder judicial, p.ej. otros jueces, presidentes de los tribunales (Sillen, 2019: 110 y ss.).

\footnotetext{
10 Sentencia A.K. (Independencia de la Cámara Disciplinaria del Tribunal Supremo), apdo. 118.

11 Sentencias Comisión/Polonia (Independencia del Tribunal Supremo), apdo. 71-73, y Comisión/Polonia (Independencia de los Tribunales Ordinarios), apdo. 109-110.

12 Sentencias Comisión/Polonia (Independencia del Tribunal Supremo), apdo. 76, y Comisión/Polonia (Independencia de los Tribunales Ordinarios), apdo. 113.
} 
Teniendo en cuenta lo anterior, vale la pena indicar que las garantías de independencia e imparcialidad -en su conjunto- postulan la existencia de reglas, especialmente en lo referente a la composición del órgano jurisdiccional, así como al nombramiento (modo de designación), a la duración del mandato y a las causas de inhibición, recusación y cese de sus miembros, al procedimiento relacionado con la jubilación y a la protección frente a las presiones (incluyendo los mecanismos de la responsabilidad disciplinaria), que permitan excluir toda duda legítima en el ánimo de los justiciables en lo que respecta a la impermeabilidad de dicho órgano frente a elementos externos y en lo que respecta a su neutralidad con respecto a los intereses en litigio ${ }^{13}$.

En lo que se refiere directamente a la edad de jubilación, el TJUE recordó la importancia del principio de inamovilidad, parte importante de la mencionada independencia judicial (de orden externo). Estas reglas exigen, en particular, que los jueces puedan permanecer en el ejercicio de sus funciones en tanto no hayan alcanzado la edad de jubilación establecida en la ley o hasta que termine su mandato, cuando éste tenga una duración determinada. Aunque estos requerimientos no tienen carácter absoluto, es necesario, en cualquier caso, que se adopten medidas adecuadas y proporcionales, como un período transitorio o un planteamiento gradual que permitan prevenir que dicha reducción se utilice de forma encubierta para modificar la composición de los órganos judiciales. En todo caso, hay que evitar cualquier impresión de que el acortamiento del período de ejercicio de la función jurisdiccional se debe en realidad a las actividades desarrolladas por estos durante el ejercicio de sus funciones ${ }^{14}$.

Según el TJUE, no se ha demostrado que las medidas objeto de controversia constituyan un medio adecuado, ni que fuera necesario imponer la jubilación forzosa de los jueces del TS. Rechazó el argumento de que la reducción de la edad de jubilación a 65 años se debiera a la voluntad de armonizarla con la edad general de jubilación. La última, en todo caso, no supone una jubilación forzosa de los trabajadores y las trabajadoras, sino meramente el derecho - una facultad- de poner fin a su actividad profesional y percibir una pensión de jubilación ${ }^{15}$. El TJUE no tenía dudas de que el motivo real de esta modificación era apartar a un determinado grupo de jueces del TS y crear un instrumento de presión y control político. En el contexto de la posibilidad de prórrogas - dos, cada una por tres años- por parte de los órganos del Poder ejecutivo, el TJUE indicó que era muy difícil defender que se tratara realmente de una armonización con la edad general de jubilación (65 años para los hombres y

13 Sentencia Comisión/Polonia (Independencia del Tribunal Supremo), apdo. 74.

14 Ibidem, apdo. 80 y ss.

15 Ibidem, apdo. 90. 
60 años para las mujeres), ya que su concesión significaba que un juez del TS, según las nuevas normas, podía seguir activo hasta los 71 años ${ }^{16}$.

En lo que se refiere a la prórroga de funciones en sí misma, el TJUE no excluye la existencia de tal mecanismo. Si bien corresponde en exclusiva a los Estados miembros decidir si la autorizan o no una vez alcanzada la edad de jubilación ordinaria, no es menos cierto que están obligados a velar por que las condiciones y las modalidades a las que se sujeta la prórroga no puedan menoscabar el principio de independencia judicial ${ }^{17}$. Además, la circunstancia de que un órgano como el Presidente de la República (Ministro de Justicia, en el caso de los tribunales ordinarios) tenga atribuida la facultad para decidir si concede o no una prórroga no basta, por sí sola, para concluir que se ha violado el principio de independencia judicial. No obstante, es importante asegurarse de que los requerimientos sustanciales y las normas de procedimiento que rigen la adopción de tales decisiones respeten la impermeabilidad de los jueces frente a elementos externos ${ }^{18}$.

En este contexto, en primer lugar, el TJUE indicó que los mecanismos de prórroga introducidos en el sistema polaco tenían carácter totalmente discrecional, por cuanto su adopción no estaba en sí sometida a ningún criterio objetivo y verificable y no debía estar motivada. Los criterios establecidos en la ley eran excesivamente vagos, por lo cual no podía verificarse su concurrencia. Además, no cabía interponer un recurso judicial contra tal decisión ${ }^{19}$. En el caso de los tribunales ordinarios la ley tampoco fijaba plazo para que el Ministro de Justicia adopte la decisión acerca de la prórroga, lo que prolongaba el período de incertidumbre ${ }^{20}$.

En segundo lugar, el TJUE mencionó que la participación del Consejo Nacional del Poder Judicial en el procedimiento de la concesión de prórroga podía objetivar todo el proceso, siempre que este órgano sea independiente de los poderes legislativo y ejecutivo ${ }^{21}$. Pero en esta esfera tampoco existía una norma que obligara al Consejo polaco a motivar sus dictámenes, por lo cual su participación no contribuía a orientar de manera objetiva al órgano ejecutivo en el ejercicio de la facultad de autorizar o denegar la prórroga. Además, la práctica indicaba que el Consejo Nacional del Po-

16 Ibidem, apdo. 83 y 85

17 Sentencias Comisión/Polonia (Independencia del Tribunal Supremo), apdo. 110, y Comisión/Polonia (Independencia de los Tribunales Ordinarios), apdo. 118.

18 Sentencias Comisión/Polonia (Independencia del Tribunal Supremo), apdo. 111, y Comisión/Polonia (Independencia de los Tribunales Ordinarios), apdo. 119

19 Sentencia Comisión/Polonia (Independencia del Tribunal Supremo), apdo. 114.

20 Sentencia Comisión/Polonia (Independencia de los Tribunales Ordinarios), apdo.123.

21 Aquí el TJUE hizo referencia al problema de la independencia del Consejo Nacional del Poder Judicial. Esta cuestión fue resuelta en la posterior sentencia A.K. (Independencia de la Cámara Disciplinaria del Tribunal Supremo), donde indicó las herramientas para evaluar la independencia de los consejos del Poder judicial y enfatizó las circunstancias que pueden suscitar dudas acerca de la independencia del Consejo polaco, apdo. 124 y 143. 
der Judicial se limitaba a emitir sus dictámenes, fueran favorables o desfavorables, a veces sin motivación alguna y en otras ocasiones con una motivación puramente formal ${ }^{22}$.

V.

Los argumentos del TJUE acerca de la reducción de la edad de jubilación y el mecanismo de prórroga son muy razonables y bien fundados. Era evidente que se trataba de instrumentos de carácter puramente político con el propósito de acabar con la independencia judicial y destituir a los jueces del TS con más experiencia. En el contexto de otros cambios en la Administración de Justicia en Polonia -sobre todo el cambio de la estructura del TS, la creación de las nuevas cámaras del TS y el asalto a la autonomía del Consejo Nacional del Poder Judicial- no cabe ninguna duda de que se trataba de la captura política de la máxima instancia judicial en Polonia. La respuesta del TJUE fue muy clara: la independencia judicial no es un asunto interno y el Derecho de la Unión proporciona instrumentos para su efectiva protección. Hay que agradecer esta postura y el esfuerzo interpretativo del TJUE, aunque, teniendo en cuenta que la manipulación de la edad de jubilación es solo una de las medidas aplicadas últimamente por el gobierno populista, la batalla por la independencia de la judicatura polaca no se ha terminado. 


\section{BIBLIOGRAFÍA}

Blokker, P. (2019). Populist Counter-Constitutionalism, Conservatism, and Legal Fundamentalism. European Constitutional Law Review, 15 (3), 519-543.

Bonelli, M. y Claes, M. (2018). Judicial serendipity: how Portuguese judges came to the rescue of the Polish judiciary: ECJ 27 February 2018, Case C-64/16, Associação Sindical dos Juízes Portugueses. European Constitutional Law Review, 14 (3), 622-643.

Brzozowski, W. (2018). The Removal of Judges and the Rule of Law. En M. Belou (Ed.), Rule of Law at the Beginning of the Twenty-First Century (pp. 275-292). Utrecht: Eleven International Publishing.

Brzozowski, W. (2019). Can the Constitutional Court Accelerate the Democratic Backsliding? Lessons from the Polish Experience. En M. Belou (Ed.), The Role of Courts in Contemporary Legal Orders (pp. 371-382). Utrecht: Eleven International Publishing.

Castillo-Ortiz, P. (2019). The Illiberal Abuse of Constitutional Courts in Europe. European Constitutional Law Review, 15 (1), 48-72.

Chang, W. (2019). Back into the political? Rethinking judicial, legal, and transnational constitutionalism. International Journal of Constitutional Law, $17(2), 456-457$.

Faggiani, V. (2019). La 'rule of law backsliding' como categoría interpretativa del declive del constitucionalismo en la UE. Revista Española de Derecho Europeo, 71, 57-100.

Garlicki, L. (2019). Constitutional Court and Politics. The Polish Crisis. En C. Landfreid. Judicial Power: How Constitutional Courts Affect Political Transformations (pp. 141-162). Cambridge: Cambridge University Press.
Krzywoń, A. (2018). La crisis constitucional en Polonia (2015-2017): Como cuestionar el sistema del equilibrio constitucional en dos años. Teoría y Realidad Constitucional, 41, 359-379.

Krzywoń, A. (2020). La defensa y el desarrollo del principio de independencia judicial en la Unión Europea. Revista Española de Derecho Constitucional, 119, 85-117.

Lautenbach, G. (2013). The Concept of the Rule of Law and the European Court of Human Rights. Oxford: Oxford University Press.

Martín y Pérez de Nanclares, J. (2017). El TJUE como actor de la constitucionalidad en el espacio jurídico europeo: la importancia del diálogo judicial leal con los tribunales constitucionales y con el TEDH. Teoría y Realidad Constitucional, 39, 235269.

Sadurski, W. (2019a). Poland's Constitutional Breakdown. Oxford: Oxford University Press.

Sillen, J. (2019). The concept of 'internal judicial independence' in the case law of the European Court of Human Rights. European Constitutional Law Review, $15(1), 110-124$.

Szente, Z. (2017). Challenging the Basic Values Problems in the Rule of Law in Hungary and the Failure of the EU to Tackle Them. En A. Jakab, D. Kochenou (eds.). The Enforcement of EU Law and Values: Ensuring Member States' Compliance (pp. 456-475). Oxford: Oxford University Press. 